http://revistaurbanismo.uchile.cl

\title{
Alternativas de gestión urbanística: Una mirada reflexiva hacia los últimos diez años.
}

Alternatives for urban management: A reflective look at the past ten years.

Miguel Rótolo

\section{Filiación}

Arquitecto de la Universidad Nacional de Mar del Plata, Argentina. 1987. Maestría en Gestión Ambiental del Desarrollo Urbano - Universidad Nacional de Mar del Plata, en curso. Docente del Área Arquitectónico - Urbanística e Investigador del Centro de Estudios para el Desarrollo Urbano de la Facultad de Arquitectura Urbanismo y Diseño. UNMdP. Miguel Rótolo: mcrotolo@copetel.com.ar mcrotolo@mdp.edu.ar

\section{Resumen}

Existe abundante literatura acerca de la arquitectura y su relación con la ciudad. Sin embargo, pocos comprenden a la arquitectura como un instrumento para construirla, y casi nadie entiende su rara mezcla de poder e impotencia. Así, la construcción de la ciudad, aparece como una compleja relación entre distintos agentes, cuyas acciones generan una metástasis en el tejido urbano. Conocer la manera de actuación de estos agentes y la organización de las políticas del Estado, permite encontrar elementos para incidir, persiguiendo efectos que redunden en beneficios para la ciudad y sociedad en general.

\section{Palabras clave}

Transformación urbana, grandes proyectos urbanos, gestiones alternativas, Mar del Plata.

\begin{abstract}
There is a lot of literature about architecture and its relation with the city, however, there are few people who understand architecture like an instrument for constructing a city, and almost nobody understands the architecture's paradoxical mixture of power and inability. So, the construction of a city appears like a complex relation among different factors, whose actions generate a metastasis in the urban frame. To know the way of these factors performance, and the organization of the State-policies, allow us to find elements to operate on its design, following effects that give advantages to the city and society in general.
\end{abstract}

\section{Key words}

Urban transformation, huge urban projets, alternative arrangements, Mar del Plata.

\section{Sumario}

Introducción

Acerca del Estado y la formación social en América Latina

El Estado en el nivel municipal

Las intervenciones en la ciudad

Los Grandes Proyectos como alternativa de intervención

La transformación de la calle Alem en Mar del Plata

Transformaciones a partir de los balnearios de La Perla en Mar del Plata

Intervenciones urbanísticas relativas a los "XII Juegos Deportivos Panamericanos. Mar del Plata '95"

Consideraciones finales respecto a los casos comparados

Reflexiones de carácter general

Bibliografía 
$\mathrm{ht \dagger p}: / /$ revistaurbanismo.uchile.cl

\section{Introducción}

La última década se ha caracterizado, en América Latina, por los cambios estructurales producidos en la economía de sus países.

El modelo de auge del capitalismo de los años de la posguerra, con crecimiento y estabilidad, con un sistema monetario y financiero estable, con reglas claras sobre la fijación de los tipos de cambio y tasas de interés, aparece sólo como referencia histórica. El denominado capitalismo posmoderno, de articulación pasiva y residual con el mercado mundial, funciona con supuestos diferentes a los del capitalismo moderno, construido inmediatamente después de la segunda guerra mundial. De aquí el origen de la crisis del modelo de pensamiento neoclásico - neokeynesiano y los límites evidentes de la ola neoliberal en su apogeo y ocaso de los 80 s y 90 s, respectivamente.

El estilo, aún vigente pero en profunda crisis, se presentó como única alternativa de solución (coyuntural), de estabilización tras el fracaso del modelo anterior, armado sobre la base de un esquema similar al modelo agro-exportador que tuvo presencia hasta la mitad del siglo pasado, modificando sensiblemente el aparato proteccionista y postulando la integración intima al mercado mundial, poniendo en un dilema incluso el concepto de nación.

Durante el auge y el declive de la ola neoliberal mencionada hemos observado un conjunto de políticas macroeconómicas que no se constituyó en lo que Khun ${ }^{1}$ llamaría un "paradigma", y tampoco en alternativa al modelo de desarrollo, permaneciendo en una política promotora de desprotección, desestatización y desregulación, fundada en el retorno a "la mano invisible del libre mercado". Sus rasgos principales consistieron en abogar por un Estado mínimo (en lo social) y subsidiario (en lo económico), que no permitió un crecimiento sostenido con empleo y bienestar social, ni se constituyó en un modelo alternativo de desarrollo para los países de América Latina provocando, en definitiva, una crítica declinación de la cosa pública en general.

En el marco de una reestructuración de alianzas entre naciones, los llamados países centrales siguieron políticas de bloque altamente proteccionistas de sus productos, en tanto que los periféricos se vieron afectados por las políticas de ajuste, que incidieron provocando una retracción no sólo del aparato productivo, sino también de aquellos sectores que por su especificidad podrían competir.

La reducción del crédito por la política restrictiva perjudica entonces a la pequeña y mediana empresa en contrapartida con lo sucedido respecto de las empresas grandes y las transnacionales, acentuando el proceso de concentración, sobre la base de la distribución desigual de la riqueza.

1 KUHN, T.S. La estructura de las revoluciones científicas. FCE. México. 1985. 


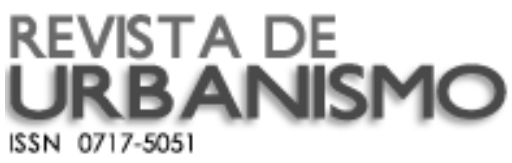

http://revistaurbanismo.uchile.cl

\section{Acerca del Estado y la formación social en América Latina}

Indudablemente, la configuración histórica de la formación social de Latinoamérica está íntimamente relacionada a la instauración del Estado.

En la mayoría de los países europeos el surgimiento del Estado ocurrió en el encuadre de sociedades previamente consolidadas, lo cual equivale a decir que se inició a partir de una necesidad, en ese caso de organización y convivencia social.

En América Latina, el Estado se trasladó impuesto (y ya armado) y precediendo a la formación de la sociedad, surgido también de una necesidad, pero en este caso como instrumento de dominación por parte de las metrópolis históricas, rasgo que, con las variantes propias de la evolución, aún conserva.

Esta característica genealógica del Estado latinoamericano ha encorsetado, históricamente el accionar de la sociedad, generando un artefacto independiente, autoritario, corporativista, atrofiado y con un gran despliegue burocrático, que no interactúa, sino más bien tiende a segregarse de la sociedad, la cual debería ser su sentido. Más bien, ha efectuado siempre una presión, desde arriba hacia abajo, con el objeto a influir en la sociedad, manteniendo su carácter dominante.

La cuestión que ha de ser notada es la sorprendente velocidad del proceso de modernización que se ha dado en América Latina, más precisamente entre mediados del siglo XIX y mediados del siglo $\mathrm{XX}$, donde aquellos procesos que maceraron lentamente en Europa conformando complejos y densos estratos, en Latinoamérica se han presentado de forma sintetizada ${ }^{2}$.

Tres aspectos merecen destacarse siguiendo a Guimaraes ${ }^{3}$ :

- El carácter burocrático y patrimonial del Estado ha condicionado, y probablemente lo siga haciendo, la constitución de la sociedad y su quehacer político; con una estructura de poder concentrada y excluyente, toma de decisiones en función de estratos más organizados (en términos de poder), con un fuerte contenido tecnocrático y formal en la resolución de conflictos sociales. En suma, una marcada idea de verticalidad.

- El carácter de desigualdad e inequidad entre los distintos estratos sociales, sumado al deterioro del medio ambiente y del patrimonio ecológico ${ }^{4}$, producto

GORELIK, Adrián, Imaginarios urbanos e imaginación urbana. Ponencia. En BazarAmericano.com

3 GUIMARAES, Roberto, "El leviatán en extinción? Notas sobre reforma del Estado en América Latina". En Revista SITUACIÓN Nro. 1. junio 1996.

4 La problemática de la desigualdad y el deterioro ambiental es también histórica. Los distintos modelos, desde la época colonial hasta la actualidad, han centrado su interés en la extracción de los recursos naturales, utilizando mano de obra local, en condiciones de explotación, y en todos los casos sin tener en cuenta los daños ocasionados en el ambiente, cuestión esta que en los primeros tiempos era desconocida, y que en la actualidad, a pesar de conocerse es dejada de lado porque produce deseconomías, siendo un 
http://revistaurbanismo.uchile.cl

de insuficiencias estructurales en las estrategias de crecimiento adoptadas históricamente, incorporando ciertos sectores, a cambio de altos costos en las mayorías y descuidando el ambiente.

- La creciente crisis operativa del Estado, en los últimos años ha devenido en una crisis de legitimidad de sus instituciones.

La última década del siglo pasado importó una trascendental arremetida contra las instituciones del Estado fundadas en la inoperatividad del mismo. Se lanzaron diatribas hirientes generando un cierto convencimiento social que abrió el camino de una reforma impuesta, basada en el desmantelamiento del aparato intervencionista de décadas anteriores (empresas y servicios públicos, estructuras de poder, etc.) trasladando estas cuestiones a manos privadas (vía contratos desfavorables), dejándolas, consecuentemente, en manos del mercado.

El rol del Estado - a partir de aquellas reformas implementadas - pasó a ser mucho más pasivo siendo, en parte, sustituido por el mercado.

En ese marco, el municipio, la instancia más cercana a la ciudad, ha debido intentar alternativas de gestión distintas de los paradigmas tradicionales, en la búsqueda de mantener la iniciativa respecto de la regulación y el control de las transformaciones urbanas.

Ahora bien, existen numerosos estudios acerca del la cuestión del Estado y, en particular, en el caso de América Latina ${ }^{5}$.

Luego de su reconocimiento queda claro que representa la relación fundamental de dominación de la sociedad capitalista. Tal posición le permite reflejar el sentido de las correlaciones de fuerzas sociales principales y hegemónicas e imponer al conjunto de la sociedad la primacía de los intereses que representa.

En consecuencia, el Estado es la expresión, por una parte, de un patrón de acumulación que alude a las características de un modo de producción determinado por la forma de su participación en la economía; y, por la otra, de un patrón de hegemonía que apunta a la relación entre sociedad civil y Estado, la cual caracteriza las formas de dominación política y de legitimidad.

obstáculo al proceso productivo o, en todo caso, eliminando las ventajas comparativas. Una visión detallada de estos procesos, desde los inicios de la colonización hasta finales del siglo pasado, puede verse en GALEANO, Eduardo, Las venas abiertas de América Latina. Siglo XXI. Buenos Aires. 1986.

5 Al respecto ver, entre otros: O'DONNELL, Guillermo, "Reflexiones sobre las tendencias generales de cambio en el estado burocrático-autoritario". Doc. CEDES/ G.E.CLACSO Nro. 1, 1975. "Apuntes para una Teoría del Estado". Doc. CEDES/ G.E.CLACSO Nro. 9, 1977. PLA, Alberto, Estado y sociedad en el pensamiento Norte y Latinoamericano. Cántaro. Bs.As. 1987. Oszlak, Oscar, La formación del Estado, Bs. As. 2002. Estado y sociedad. Nuevas reglas de juego? En www.clad.org.ve. 
http://revistaurbanismo.uchile.cl

Considerar al Estado como el centro de las decisiones políticas y administrativas permite suponer una gran capacidad de mediación, de autonomía relativa decisional y de instrumentación, características que se modifican en sus niveles y magnitud, de acuerdo con las condiciones históricas.

\section{EI Estado en el nivel municipal}

Hemos puesto de manifiesto nuestra visión acerca del Estado, (sus orígenes y conformación en América Latina), pero si nos centramos en la problemática urbana (es decir la de la ciudad) debemos hacer referencia al Municipio, al Estado en el nivel más próximo a la ciudad, el nivel local.

Cabe distinguir tres contenidos (dimensiones integradas) del término municipio:

- como ámbito territorial, un segmento del territorio provincial legalmente establecido, en el que ejerce cierta competencia una Municipalidad.

- como ámbito estatal, el nivel más descentralizado del Estado, el más ligado a la vida cotidiana de la población, aquel que posibilita una gestión más ligada a la realidad de la sociedad local.

- como sociedad local, el conjunto de agentes y de relaciones sociales, económicas y políticas que se dan dentro de un ámbito territorial determinado.

El municipio es la organización del gobierno que corresponde a la ciudad. En tanto gobierno local, y como parte del Estado, es parte de una correlación de fuerzas sociales locales que se redefine en el tiempo (a lo largo de la historia) y en el territorio; esa redefinición está ligada a su función de servir a la organización económica de la producción y la reproducción de la población, sea a través del ámbito público o del privado.

La ciudad, en tal sentido, es el producto de una interacción de clases sociales, grupos de interés e individuos. Cada unidad tiene o adquiere un significado particular que resulta de la definición del conflicto a través del cual los intereses dominantes imponen sus objetivos y su imagen al sistema urbano. Así es entendida como parte de una noción dinámica, que se modifica con el tiempo con el producto de la puja de los intereses sociales.

Más allá de formulaciones teóricas, en los hechos el cumplimiento del papel local del Estado no está solo a cargo del municipio. Más bien parecería que se produce una suerte de competencia inter - jurisdiccional en la que intervienen, además del municipio, organismos de los niveles gubernamentales centrales e intermedios.

La autonomía municipal, a su turno, puede definirse como la capacidad de la sociedad local de regir por si misma la vida institucional. 


\section{REVITADE \\ ISSN $0717-5051$}

http://revistaurbanismo.uchile.cl

En ese sentido pueden utilizarse tres indicadores:

- la capacidad de darse sus propias normas fundamentales (cartas municipales).

- la capacidad de elegir las autoridades locales. Y

- la capacidad de formular y ejecutar políticas propias.

Vemos que los municipios, por una parte, tienen facultades normativas orientadas a la regulación de la producción del suelo urbano y de las construcciones que se realizan, pautando de esa forma las acciones privadas y públicas en la ciudad; por otra parte, están a cargo de la construcción de buena parte de la infraestructura y el equipamiento urbanos, así como de la prestación de ciertos servicios.

La organización municipal del Partido de Gral. Pueyrredón, que usaremos como caso demostrativo, no escapa al patrón general organizativo de los municipios de América Latina. Posee un órgano unipersonal que tiene a cargo las funciones propiamente ejecutivas, y un órgano colegiado que tiene a su cargo funciones de tipo legislativo, (aunque las normas emanadas no tengan el carácter de leyes) y al cual se le asignan funciones de contralor respecto del ejecutivo y participa como poder en varios actos de gobierno (por ejemplo, en lo relativo a ingresos y gastos municipales).

\section{Las intervenciones en la ciudad}

En línea con nuestro enfoque, el estudio de las transformaciones urbanas, entendidas como parte de un proceso más general - el de apropiación social de la naturaleza -, debe abordarse, en principio, a partir del análisis de los agentes intervinientes, sean estos públicos o privados, y en particular del Estado.

La instancia estatal desarrolla su acción de dos modos: por un lado, efectúa una intervención directa, a partir de la programación de infraestructura y equipamiento público, mediante los cuales produce o estructura el espacio; y por otra, hace efectiva la intervención indirecta, jurídica, mediante la orientación y control de los actores privados ${ }^{6}$.

Además, debe temerse en cuenta la constante (y creciente) crítica a los modelos clásicos de planificación, surgida de la impotencia de los instrumentos tradicionales de la política urbanística, ante la atomización y fragmentación de intereses, la lucha entre grupos corporativos y la tendencia a la burocratización de la gestión urbana y social.

Los instrumentos básicos de regulación y control (Código de Ordenamiento y Código de Edificación) que, hoy por hoy, son los articuladores del urbanismo y la arquitectura y manifiestan altos grados de inoperancia y en definitiva terminan modificándose como producto de las presiones ejercidas por la realidad misma.

6 CIGNOLI, Alberto L., "Intervenciones en el Espacio Territorial". En Arquitectura No 8 año 5, ASAMP, Mar del Plata.1987.

Revista de Urbanismo N9 - Marzo de 2004 
http://revistaurbanismo.uchile.cl

Otra lección se constituye en la experiencia urbana europea en el cambio de década, caracterizada fundamentalmente por los Grandes Proyectos de Intervención Urbana, inversiones con criterio proyectual sobre partes completas de ciudad, entendiendo su construcción de una nueva manera. Se consigue, en este cometido, una redefinición de la estructura urbana en términos mucho más operativos e inmediatos que los que puede esperarse de los planes generales (de sesgo tradicional).

Tanto es así que últimamente se ha abierto una fuerte discusión en torno a los instrumentos de control urbano (más aún, abarca en general a todo control ejercido por el Estado).

En los últimos años tanto desde la teoría como desde la práctica se han lanzado críticas humillantes contra los planes urbanísticos, su abstracción cuantitativa, el papel decisivo que adquiere en ellos la normativa y las fórmulas legales y de gestión por encima de las definiciones de forma y contenido y de las decisiones puntuales, por encima de los proyectos urbanos y del valor arquitectónico de la ciudad.

Obviamente alrededor de estas reflexiones comenzaron a aparecer los antiguos enemigos de la planificación, trayendo propuestas en favor de una ciudad sin plan (con un mínimo de pautas concretas y, para lo restante, libertad total), defendiendo una visión particularista e individualizada, sin objetivos colectivos $y$, por tanto, neoespeculativa.

Como agravante, comenzó a manifestarse una cierta generización de la ciudad producto (o reflejo) de la globalización económica que, de acuerdo con Koolhaas, conlleva una homogeneización de la ciudad, una convergencia "sólo posible a costa del despojo de la identidad"7".

7 KOOLHAAS, Rem, "La ciudad genérica". En Dossier Ciudad. Beta_test/e.zine sobre arte, tecnología y medios. 2002. Si bien su perspectiva es central, sus consideraciones no resultan equivocadas respecto de las ciudades (esencialmente las grandes ciudades) latinoamericanas. 


\section{REVISTA DE \\ URBANISMO \\ ISSN $0717-5051$} Alternatives for urban management: A reflective look at the past ten years.

http://revistaurbanismo.uchile.cl
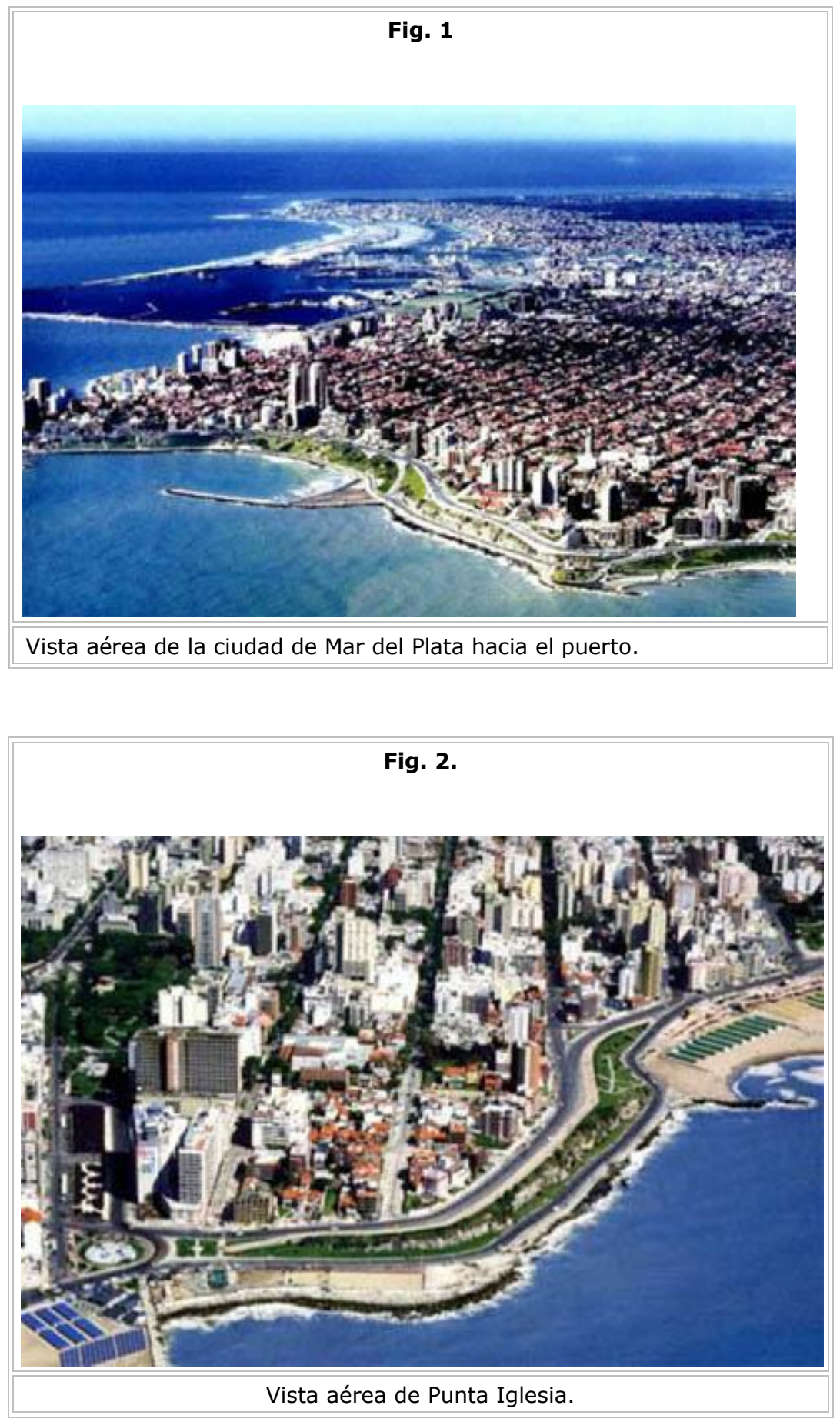


\section{Los Grandes Proyectos como alternativa de intervención}

Para la realización el presente trabajo hemos desandado el camino de ciertos Grandes Proyectos de Transformación urbana, con el objeto de indagar sus procesos de gestión, así como sus efectos.

Tres afirmaciones guiaron nuestra tarea:

- La transformación de la ciudad donde se implanta un Gran Proyecto Urbanístico resulta de la interacción de un conjunto heterogéneo y complejo de procesos desatados a partir de una decisión (política) estatal, acompañada por una serie de intervenciones de actores privados, por lo que podemos considerarla resultante de esa acción conjunta.

- A cada caso analizado le corresponde un contexto específico (singular) y una política del Estado acorde a la coyuntura.

- La forma y estructuración de las políticas del Estado (para intervenir en un sector de ciudad) se relacionan directamente con sus efectos, por lo que desde su diseño pueden preverse los beneficios (y perjuicios) que se obtendrán.

La primera afirmación pone de manifiesto la acción conjunta de los actores público y privado, en un emprendimiento (Grandes Proyectos) que pretende atender a un acontecimiento singular (por Ej., Juegos Panamericanos), a partir de la cual resulta posible influir (transformando) de algún modo sobre grandes sectores de ciudad.

La segunda, más general con relación al alcance del trabajo, define la situación particular de cada uno de los casos que serán analizados, tanto los paradigmas europeos como el caso específico de Mar del Plata y pretende enfocar el estudio de las acciones públicas en todos ellos.

Por último, la tercera, señala la relación existente entre el resultado (impacto) de los Grandes Proyectos en su entorno (mediato e inmediato) y los procesos de generación y concreción de los mismos, dejando ver que ya desde el inicio podemos ir comprendiendo a qué resultado se arribará y, por lo tanto, orientando en uno u otro sentido las acciones realizadas, valiéndonos de los instrumentos con que cuenta el Estado para estas ocasiones.

Observamos que la planificación desde el Estado ha venido proporcionando proverbialmente, en pos de objetivos y metas de bien común y de mejoramiento de las condiciones de vida de la sociedad o de un sector de esta ${ }^{8}{ }^{[8]}$ : una intervención de alguna de sus jurisdicciones (nacional, provincial, municipal) desde algún área

8 ROBIROSA, Mario y otros, Turbulencia y Planificación Social. Buenos Aires. Unicef/Siglo XXI, 1990. 
pertinente del órgano ejecutivo correspondiente; con los recursos propios, de diversa naturaleza (humanos, naturales, financieros) que tuviese asignados a dichas áreas; con la autoridad e instrumentos de que dispone el Estado para inducir controlar y/o restringir la conducta de los actores privados.

Por otro lado, ciertas conclusiones obtenidas de un trabajo anterior ${ }^{9}$, nos remitieron a los grandes proyectos de transformación urbana, que constituyen uno de los rasgos más característicos de la reciente experiencia urbana europea ${ }^{10}$. De modo aclaratorio citemos los acontecimientos singulares de Barcelona ${ }^{11}$ y Sevilla en 1992; la Exposición Internacional de Arquitectura de Berlín; así como la remodelación de los Docklands o el proyecto para King's Cross en Londres; Bicocca en Milán; Lingotto y Spina Centrale en Turín; Kop van Zuid en Rotterdam; los centros de Amiens, Aix-en-Provence o la Remodelación Urbana en Viena.

En Argentina podemos citar las intervenciones en el sector de Puerto Madero (en Buenos Aires) y las Instalaciones Básicas para los Juegos Panamericanos 1995 (en Mar del Plata).

Este último caso, con los vaivenes propios de un acontecimiento de aquella naturaleza (incluido un cambio en la conducción del municipio, a escaso tiempo de su realización), nos presenta una alternativa de intervenir con una serie de Grandes Proyectos Urbanísticos en distintos sectores de la ciudad.

Al momento de decidir cuáles serían los casos a estudiar planteamos que estos casos de urbanismo estratégico, basados en actuaciones puntuales que por su especial situación tienen el papel de un foco de regeneración expansiva (efecto de difusión espacial de innovaciones) con la particularidad de que cada uno de ellos está relacionado con un evento de relevancia internacional; léase Juegos Olímpicos 1992, Barcelona; Exposición Internacional 1992, Sevilla; IBA (Internacionale Bauausstellung), Berlín; etc.

Esta selección a partir de una causa generatriz común permitió la comparación con nuestro caso particular: Juegos Panamericanos 1995, Mar del Plata; pero a su vez planteó la cuestión de situaciones diferenciales: políticas, jurídicas, económicas, sociales y de relaciones internacionales.

9 ROTOLO, Miguel, "Procesos de Transformación Urbana. Un aporte a las estrategias de regulación en las intervenciones urbanísticas". En I+A / Investigación + Acción. FAU - UNMdP. Mar del Plata, 1994.

10 ESQUIAGA, José María, "Entre el plan y el proyecto". En A\&V (Monografías de Arquitectura y Vivienda) Nro. 30. Madrid. 1991.

11 Ver al respecto: Martorell/Bohigas/Mackay/Puigdomenech Transformación de un Frente Marítimo. Barcelona. La villa Olimpica. Barcelona. G.Gilli. 1988.

Además Martorell/Bohigas/Mackay/Puigdomenech La villa Olímpica. Arquitectura. Parques. Puerto Deportivo. Barcelona. G.Gilli. 1991. 


\section{REVISTA DE URBANISMO

http://revistaurbanismo.uchile.cl

Con estos criterios, y luego de un conocimiento preliminar de todos los paradigmas se decidió focalizar el análisis en:

- La transformación en la calle Alem, en Mar del Plata, como paradigma de confrontación, el estudio de una alternativa de intervención posterior del Estado, en forma indirecta, influyendo (jurídicamente) en la acción de los actores privados.

- Los balnearios de La Perla. Mar del Plata, analizado como un emprendimiento mixto (público-privado), a partir de una decisión del municipio, realizado con anterioridad al paradigma español.

- Juegos Deportivos Panamericanos. Mar del Plata 1995, como el caso más cercano, particularmente lo referido a la concentración de actividades (y su correspondiente asentamiento espacial), situado en el Parque de los Deportes de la ciudad.

\section{La transformación de la calle Alem en Mar del Plata}

En el caso correspondiente al proceso de transformación de la calle Alem, entendido como producto de la concurrencia de acciones de actores privados sin previa organización, hemos realizado un estudio desde su estado inicial (1980), en términos físicos:

Se dedujeron de este análisis de la evolución (transformación) algunas conclusiones que expresan el reflejo de un proceso acontecido en el sector, que fue originado y producido por los distintos actores que intervinieron en su construcción. En este sentido, se revisó conjuntamente al estudio de dichos actores, la intervención del Municipio (intervención indirecta, en este caso). Para ello se examinaron, y compararon, las distintas modificaciones que sufrió el Código de Ordenamiento Territorial del Partido de Gral. Pueyrredón. Observada la plancheta que corresponde al sector en cuestión, presenta diez modificaciones desde su primera redacción en el año 1979.

Luego de estudiado el listado de Ordenanzas Municipales que hacen al control, por parte del Municipio, del crecimiento y modificación del sector, nos detuvimos por un momento a analizar una instancia más general de dicho control estatal, es decir la jurisdicción provincial.

Por su parte, la Provincia de Buenos Aires sancionó y promulgó con fuerza de Ley de Ordenamiento Territorial y Uso del Suelo (8.912) en octubre de 1977 (época del denominado Proceso de Reorganización Nacional, con el Gobernador de la Provincia en ejercicio de las facultades legislativas). Queda a cargo de su implementación el Ministerio de Obras y Servicios Públicos de la Pcia. de Buenos Aires.

La misma menciona entre los objetivos del ordenamiento territorial: 
http://revistaurbanismo.uchile.cl

f) Posibilitar la participación orgánica de la comunidad en el proceso de ordenamiento territorial, como medio de asegurar que tanto a nivel de la formulación de propuesta, como de su realización se procure satisfacer sus intereses, aspiraciones y necesidades.

g) Propiciar y estimular la generación de una clara conciencia comunitaria sobre la necesidad vital de la preservación y recuperación de los valores ambientales.

Resulta curioso el análisis de los objetivos anteriormente citados, pues si bien aparecen como un conjunto de expresiones declamatorias vemos como contradictorio la procedencia de un gobierno autoritario, los cuales ponen en valor la participación y la conciencia comunitaria.

Claro está que cada una de estas expresiones no dejan margen de discusión, pues presentan un amplio grado de generalidad, y además la imposibilidad de no acordar con ellas. Sin embargo puede afirmarse que son condiciones necesarias pero no suficientes a los efectos de llevar adelante un real ordenamiento del territorio, ya que a la vista está (luego de la observación del espacio urbano) que las fallas se encuentran en la implementación en la cual, muchas veces, no han sido tenidas en cuenta, por lo menos en forma prioritaria.

Detectamos de la observación in situ, por un lado, edificaciones en altura (que, a priori, transgreden el actual Código de Ordenamiento Territorial) con basamento de uso comercial las cuales sugieren acciones de actores privados con intereses particulares. Por otro, descubrimos a los propietarios de la tierra en el sector (incluso de las construcciones) quienes a pesar de no haber sido objeto específico de nuestro estudio, nos permiten formular la hipótesis de dos grupos con intereses encontrados: quienes se ven beneficiados con la obtención de una renta diferencial debido a la utilización de su vivienda y/o terreno con fines comerciales, ya no para su uso específico originario, y quienes en una zona adyacente se ven perjudicados por las perturbaciones ocasionadas por la nueva actividad en un área destinada a la residencia.

Esta hipótesis nos remitió al estudio de las variaciones del valor del suelo en el sector, para lo cual se realizó el análisis sobre parcelas-testigo ubicadas en forma directa sobre la calle Alem y el de otras situadas en calles adyacentes.

El estudio realizado nos ha permitido demostrar el sensible incremento de los valores del suelo en el sector a lo largo del proceso de transformación. 


\section{REVISTA DE \\ URBANISMO \\ ISSN 0717-5051}

http://revistaurbanismo.uchile.cl

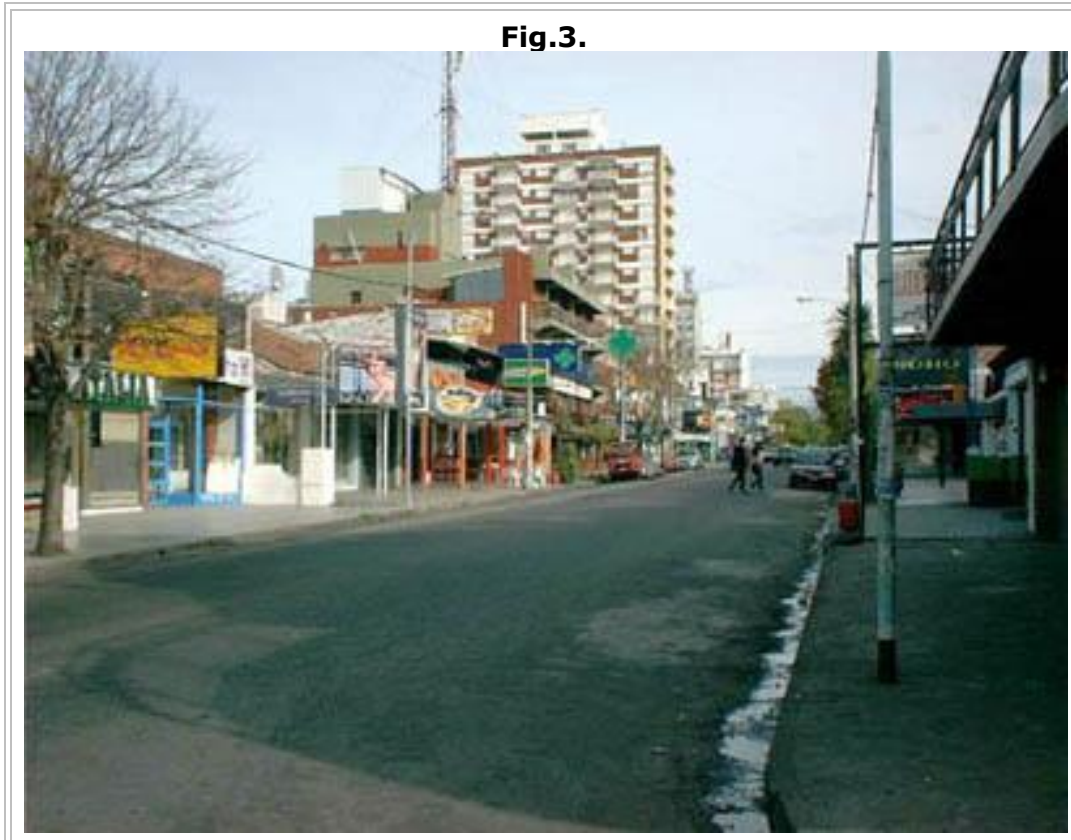

La calle Alem 2003.

Fig.4.

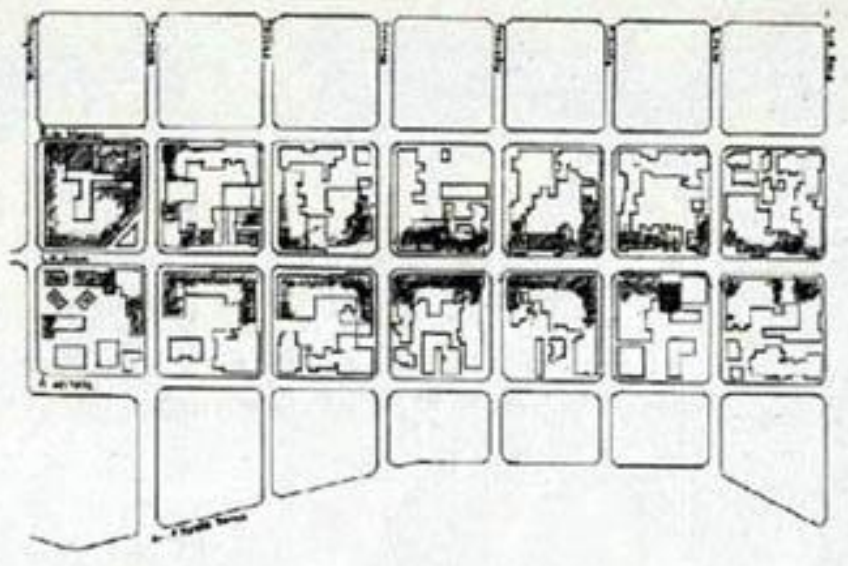

Restitución fotográfica y ocupación comercial en 1992. 


\section{REVITADE}

the past ten years.

http://revistaurbanismo.uchile.cl

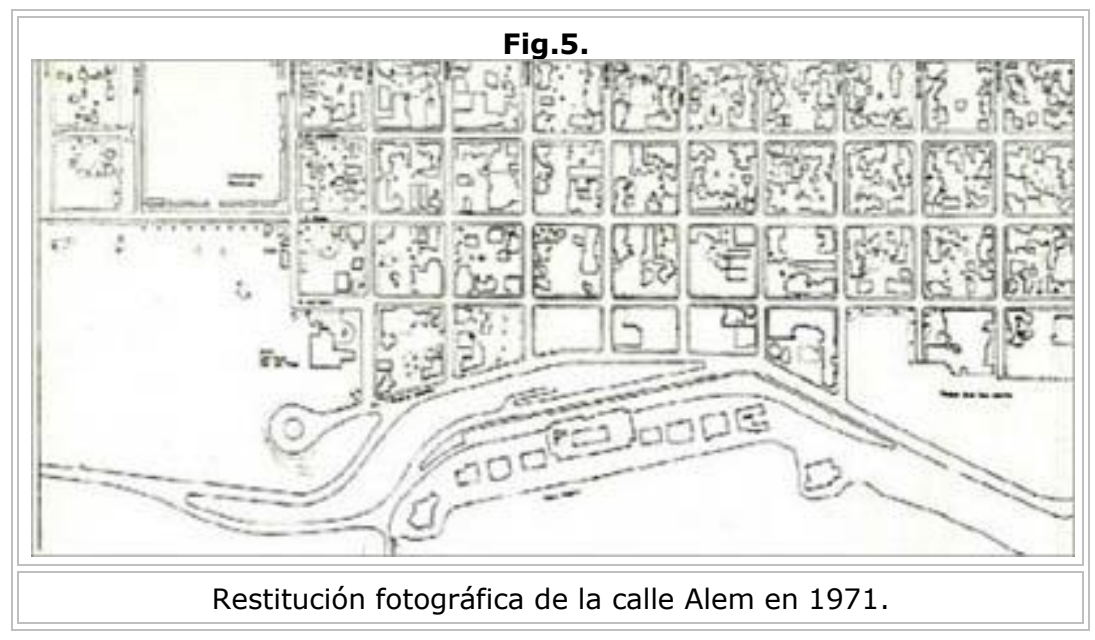

\section{Transformaciones a partir de los balnearios de La Perla en Mar del Plata}

En otro de los sectores que nos ocupó, que corresponde a los balnearios de La Perla, construido a partir de una intervención directa del Municipio, las cuestiones a tener en cuenta nos dirigieron a una distinta modalidad de análisis.

En cuanto al estado inicial y los antecedentes del sitio, se presenta como un sector que por su centralidad e importancia, arrastraba un déficit casi crónico de servicios propios de la actividad turística, una zona balnearia cuyos usuarios son en gran parte habitantes permanentes de la ciudad.

Pese a su cercanía respecto del centro, observamos un corte en el continuo de la costa, dentro del ámbito urbano, y al igual que en el sector de Alem, existe una importante diferenciación en el uso si es analizado en invierno/verano, problemática estructural de la ciudad de Mar del Plata.

Los citados aspectos, todos ellos relacionados, llevaron en su momento al Municipio a tomar la decisión de realizar una intervención en el sector, que en este caso como agente promotor, explicitó los objetivos de la intervención, manifestando en forma general acreditar los recursos turísticos de la ciudad y el Partido, complementando y perfeccionando los servicios al usuario para los próximos veinte años.

La iniciativa oficial permitió ver como peculiaridad, en los términos tradicionales de este tipo de eventos, al sistema de realización de la obra. En este caso, el llamado a Licitación Pública de Concesiones y Explotación, dejó al adjudicatario en el rol de comitente en relación a la concreción de la obra. 
http://revistaurbanismo.uchile.cl

El análisis de una obra impulsada por una decisión estructural del Municipio requirió ser abordado desde una óptica sensiblemente distinta al caso que nos ocupó anteriormente. Aparece aquí una complicada red de actores intervinientes en distintos niveles, con intereses particulares, sectoriales y comunitarios.

Se presenta una iniciativa que surge de la decisión del Municipio, quien consideró que el servicio al turismo no era lo suficientemente completo, debía perfeccionarse, y detectó, además, un desequilibrio entre lo privilegiado del sector de balnearios de La Perla y la obsolescencia y precariedad de las instalaciones existentes.

El Municipio planteó la escasez de sus recursos para encarar por sí una obra de utilidad pública de tal magnitud, por lo cual propuso una característica sui-generis para llevar a cabo la misma. Hizo un llamamiento a la iniciativa privada, la cual luego de realizado el Concurso de Anteproyectos en el cual la Municipalidad actuó en el rol de comitente transitorio, tomó a su cargo la construcción de la obra tras un llamado a licitación pública de concesiones y explotación.

Este sistema transfirió la persona del primer comitente (Municipalidad), al adjudicatario de las concesiones turísticas respectivas, para el proyecto y la dirección de las obras.

El interés del Municipio se puso de manifiesto al tomar una iniciativa de este tipo, tras reconocer las limitaciones de la coyuntura. Por esto se adoptó una modalidad alternativa de concreción de obra pública, modificando la estrategia habitual.

Una toma de decisión de estas características permitió suponer una priorización, por parte del Estado, respecto de la actividad turística local, la cual se verá, finalmente, reflejada en un mejoramiento de las condiciones de vida para la población toda.

Para este caso el Municipio fijó las reglas del juego, en dos instancias distintas: a) en lo que hace al proyecto arquitectónico a través de las Bases del Concurso de Anteproyectos y b) en lo que hace a la concesión de los balnearios a partir del Pliego de Bases y Condiciones para la Licitación Pública de Unidades Turísticas Fiscales.

Afirmábamos para el caso de la calle Alem que las distintas intervenciones de los actores privados a lo largo del período estudiado produjeron un incremento notorio en los precios, y por consiguiente elevaron la renta. Realizado el mismo análisis para terrenos ubicados en relación al Complejo Balneario La Perla las conclusiones obtenidas son sensiblemente distintas.

Observamos que no existió variación significativa en el valor de la propiedad, en el sector en estudio, desde el inicio de las obras del complejo La Perla hasta la fecha de su conclusión. La diferencia amplia que se percibió entre el año 1980 y las otras muestras ya había sido apreciada en el estudio de la calle Alem, resultando evidente 


\section{REVISTA DE \\ URBANISMO \\ ISSN 0717-5051} the past ten years.

http://revistaurbanismo.uchile.cl

que se debe a otros factores (de economía general) y no a las causas que interesan al presente trabajo.

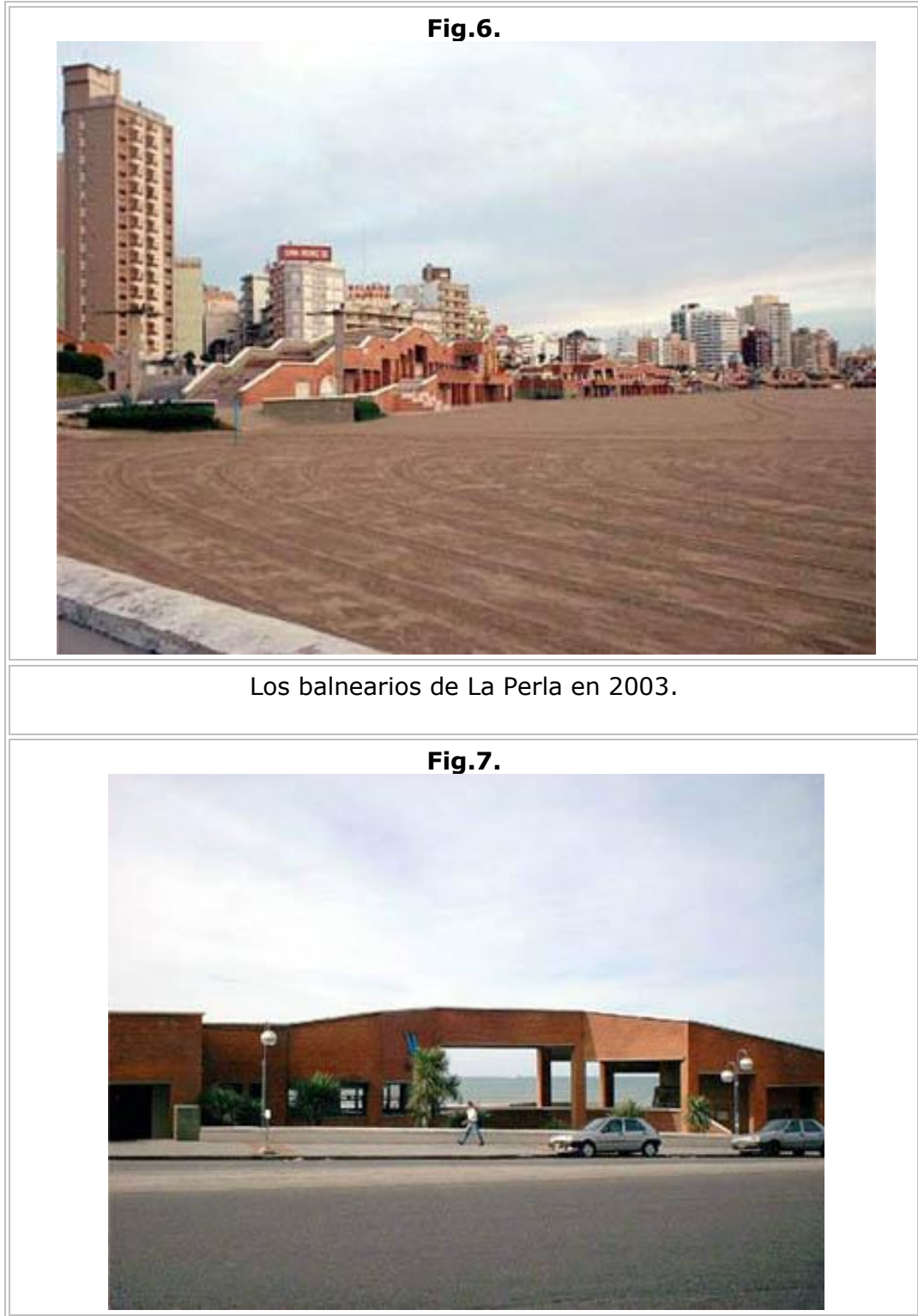

La ventana al mar desde la calle Balcarce. 


\section{REVISTA DE \\ URBANISMO \\ ISSN 0717-5051}

Alternatives for urban management: A reflective look at the past ten years.

$\mathrm{ht \dagger p}: / /$ revistaurbanismo.uchile.cl

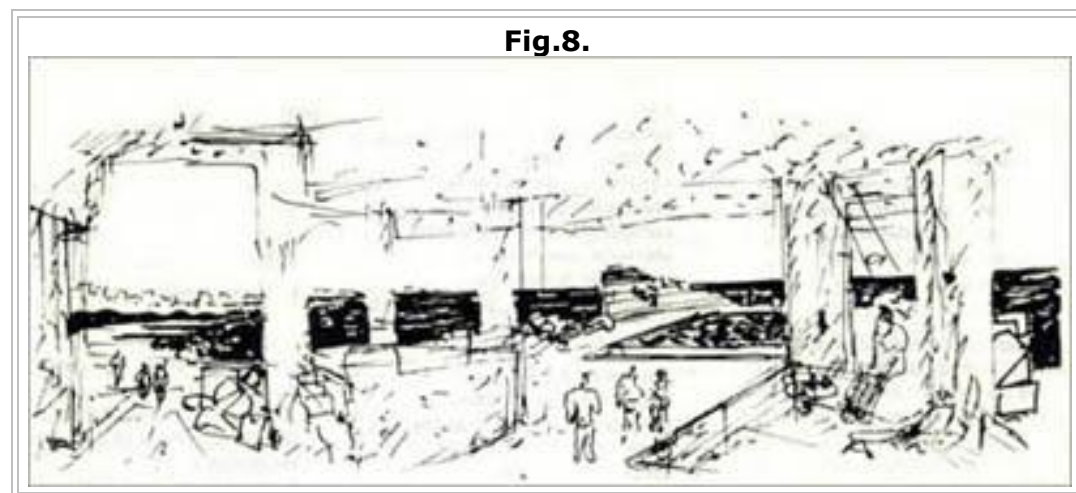

La ventana tal como la imaginó Clorindo Testa

Fig.9.

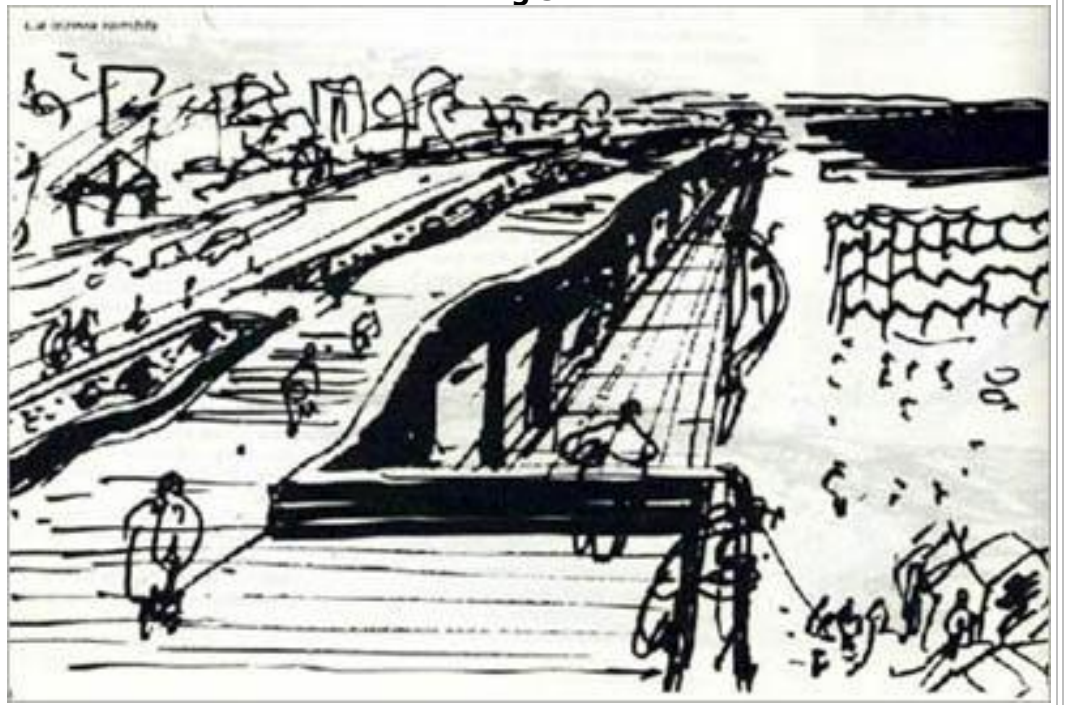

El paseo superior, en anteproyecto dibujado por Clorindo Testa. 
http://revistaurbanismo.uchile.cl

\section{Intervenciones urbanísticas relativas a los "XII Juegos Deportivos Panamericanos. Mar del Plata '95"}

Evidentemente, desde hace algunos años, las intervenciones estructurales del Estado (en el ámbito urbano), de las cuales surge la posibilidad de obtener beneficios potenciales, incorporan necesariamente el aporte de capitales privados, conformando emprendimientos mixtos (público-privado) con un objetivo determinante: la disminución de la inversión por parte del Estado, aún resignando los beneficios posteriores.

Esta concepción, que en Mar del Plata quedó de manifiesto en el caso de los Balnearios de La Perla ${ }^{12}$, fundamentada en la necesidad de ahorro por parte del Municipio, es adoptada también para la concreción de las obras destinadas a los Juegos Deportivos Panamericanos.

En la primera etapa de los trabajos -que duró hasta mediados de 1993, cuando se constituyó la Sociedad de Estado Copan '95- se esbozaron alternativas desde luego más ambiciosas que lo que finalmente se materializó: el perfeccionamiento y remozamiento del Parque Municipal de los Deportes (el viejo predio deportivo de los años 40, aderezado con el Estadio de Fútbol para el Mundial '78).

Si bien, según la Dirección de infraestructura del COPAN '95 (Comité Organizador de los Juegos Panamericanos), se realizaron 93 obras con motivo de los Juegos, entre ellas, a los efectos de nuestro trabajo, cobran importancia las que se llevaron a cabo en el Parque de los Deportes, que concentró los escenarios de los deportes más populares y que por consiguiente recibieron la mayor afluencia de público.

El predio analizado, ubicado en el cruce de la Av. Juan B. Justo y la Av. Independencia había recibido en la década del '70 las instalaciones de uno de los nuevos estadios de fútbol para el Torneo Mundial '78. En el proyecto de entonces, se había dispuesto una calle peatonal que arrancando de la intersección mencionada, llegaba, en el fondo del predio a una platea de acceso al estadio. Este eje resultó ser en 1994 el elemento vertebral del conjunto al que convergen las nuevas obras: el Estadio Polideportivo, el Complejo Ciclismo - Hockey, el Complejo Natatorio y las obras recicladas: el Patinódromo y la Cancha de Atletismo. Si se compara con el Monjuich de Barcelona, el Parque Deportivo de Mar del Plata, resulta un emprendimiento mucho menos ambicioso, lo cual se corresponde con una coyuntura económica poco propicia para "gastos de supuesto tenor superfluo"13.

La idea de conjunto aparece aquí a partir del esfuerzo (individual) de los proyectistas, quienes no recibieron otra cosa que sublotes donde se ubicarían los futuros edificios.

12 ROTOLO, Miguel (Op. Cit.).
13 FERNANDEZ, Roberto, "Realismo fin de siglo". En Revista Summa + Nro. 13. Buenos Aires. 1995. 


\section{REVISTA DE \\ URBANISMO \\ ISSN 0717-5051} the past ten years.

http://revistaurbanismo.uchile.cl

Lo cierto es que en este se presentaron varias realidades adversas:

- El desinterés de los gobiernos Nacional y Provincial por proyectos de estas características. En consecuencia el escaso apoyo político prestado al emprendimiento.

- La estrechez financiera unida a una escasa propensión por explorar financiamientos alternativos.

- La falta de una tradición político-técnica en cuanto a criterios para el cambio de la ciudad y la redefinición de sus roles regional y nacional.

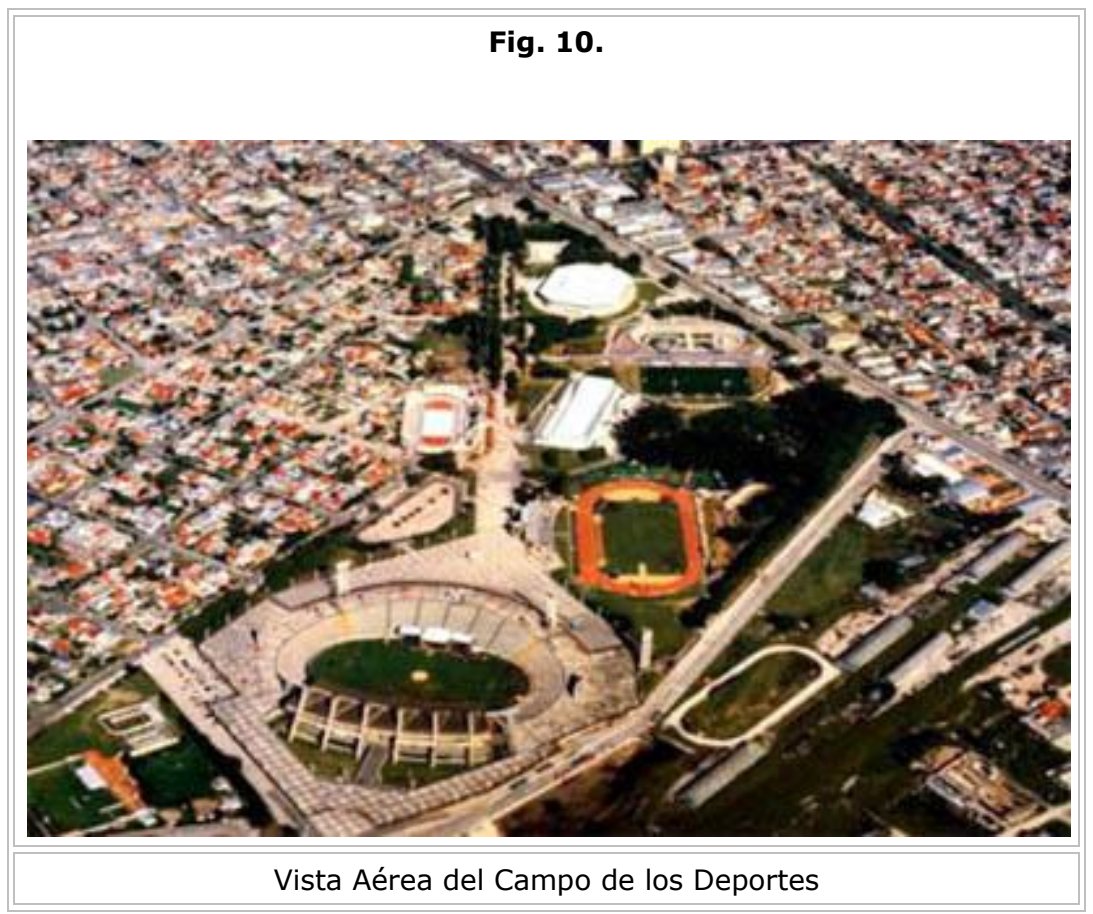




\section{REVISTA DE \\ URBANISMO \\ ISSN $0717-5051$}

the past ten years.

http://revistaurbanismo.uchile.cl

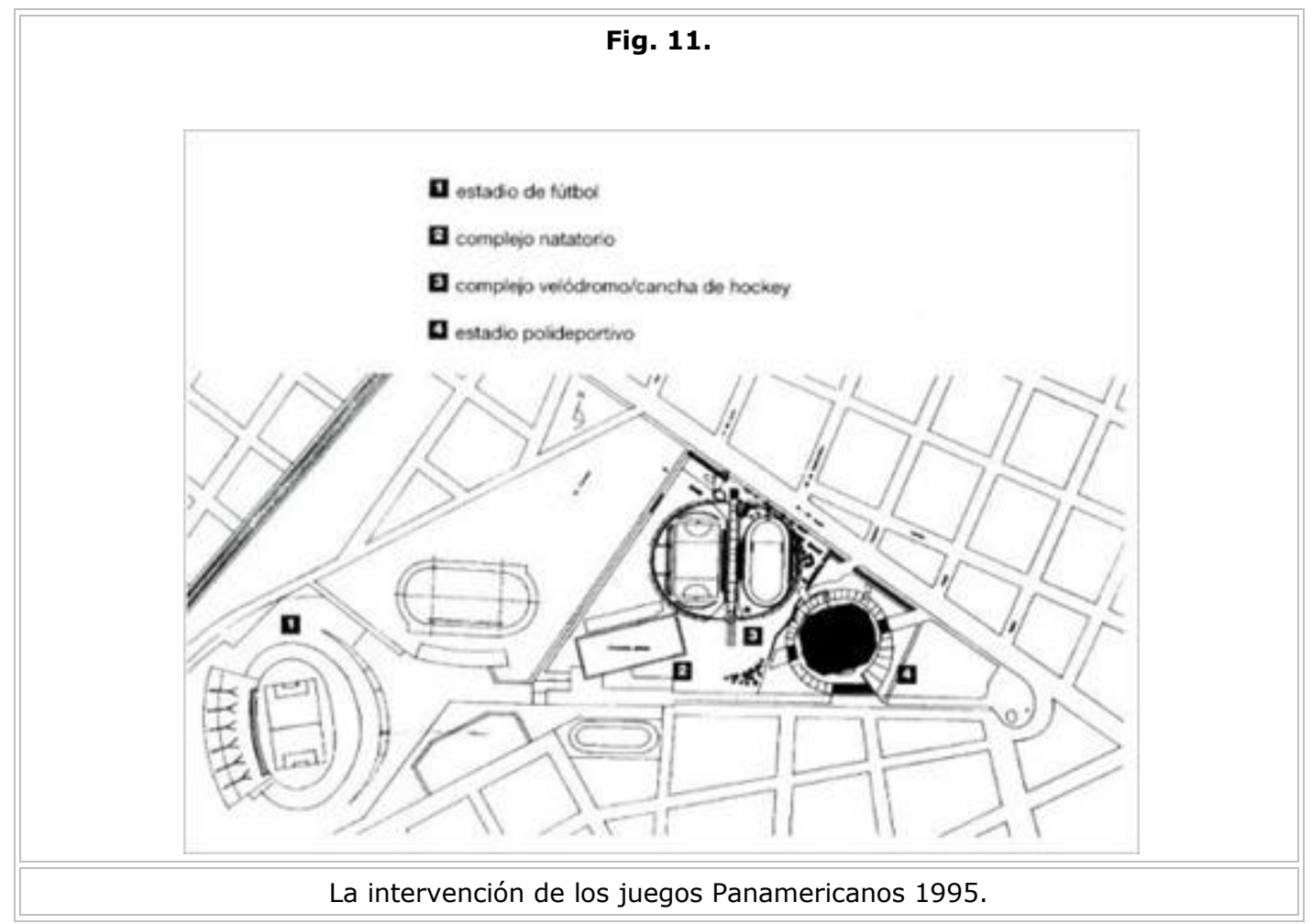

\section{Consideraciones finales respecto a los casos comparados}

Tal como fue planteado en las hipótesis de trabajo, el estudio de los casos seleccionados se orientó hacia el análisis del comportamiento de los actores sociales involucrados en el origen y la posterior implementación de decisiones estatales. En este sentido, resulta apropiado marcar las diferencias.

A) En el caso de la calle Alem, se comprobó como la posibilidad de obtener rentas diferenciales (significativas en este caso) generó un proceso de transformación en los usos de suelo urbano.

El proceso desarrollado en este sector mostró como, en un lapso relativamente corto, el uso dominante pasó de residencial a comercial, lo que desde la perspectiva de la ecología urbana podría entenderse como un proceso de invasión-sucesión. Esta 
modificación se vio materializada mediante la posibilidad de refuncionalizar el parque inmobiliario existente (antiguas viviendas, en general de gran superficie) disminuyendo la inversión inicial y en algunos casos, optimizar la rentabilidad. Los resultados obtenidos del estudio comparativo realizado sobre los valores del suelo, confirmaron esa suposición ya que el pase de un uso residencial a uno comercial repercutió notablemente en el valor del suelo y actuó como realimentador del proceso.

La caracterización de los distintos actores involucrados en este proceso demostró que sus intereses particulares aparecen materializados en el ámbito urbano (con forma y contenido), lo que permitiría al sector público incidir en los productos finales a partir de la rearticulación de las relaciones que entre ellos se establecen.

La ausencia de dicha articulación provocó, además, la abrupta diferenciación en cuanto a la intensidad de uso del sector entre la temporada turística y el resto del año.

El análisis del texto de la ley provincial 8912, de Ordenamiento Territorial, motivado por el estudio de este caso, nos llevó a concluir que los objetivos y principios que la sustentan, a pesar de ser formulada en un período autoritario, generan un marco normativo digno de ser respetado. En él se plantea la necesidad de acciones coordinadas entre la Provincia y las comunas, otorgando a éstas participación central, tanto en las decisiones de intervención directa como en las normas de regulación. Por lo tanto y teniendo en cuenta lo arduo que resultaría consensuar una nueva ley, además del tiempo que requeriría, nada impide aplicar el marco de la ley 8912 en el ámbito municipal.

El examen de las sucesivas modificaciones realizadas en el Código de Ordenamiento Territorial de la Municipalidad de General Pueyrredón, en particular en el sector estudiado, pone en evidencia que aquellas no hacen sino seguir los procesos espontáneos que se producen en el tejido urbano. Ello conlleva a que el COT, en la práctica, resulte un conjunto secuencial de normas para casos específicos. Así conviven en una misma calle edificios de una planta con torres de gran altura, construidos en su momento con normas del COT, que resultan de arduos trámites de excepción.

B) En el caso de los balnearios de La Perla, la convicción de que la actividad turística constituía la más importante fuente de recursos de la ciudad, motivó al Municipio a perfeccionar los servicios de ese carácter, postura a la puede adscribirse.

La decisión surgió como consecuencia de un amplio debate, ya que la propuesta del Departamento Ejecutivo, fue estudiada, modificada y finalmente aprobada por el Concejo Deliberante.

El método empleado para la concreción del proyecto resultó ejemplar para el abordaje de obras de magnitud, que generen beneficios para la ciudad y cuya financiación no recaiga totalmente en el Municipio. Los traspiés, que demoraron los procesos de adjudicación y de realización de la obra, se debieron a falta de experiencia en 
http://revistaurbanismo.uchile.cl

gestiones de estas características. Ya materializada en gran proporción, evidencia la mejora que acarreó en los servicios prestados a los usuarios.

Cabe observar que la forma de participación, tanto de los actores públicos como privados en la definición del proyecto, excluyó a los futuros concesionarios y que es posible la participación de éstos en la gestión de la propuesta, como de hacho se obró en la ciudad de Buenos Aires en este tipo de emprendimientos.

Resulta pertinente también señalar que distintos sitios de la ciudad, en especial los espacios litorales, son de dominio de la Provincia de Buenos Aires, lo cual origina conflictos de jurisdicción y acciones contradictorias. Este fue el caso del balneario La Perla y la experiencia plantea la necesidad de entender al espacio urbano como un todo, por sobre delimitaciones jurisdiccionales y decisiones extra-comunales que chocan con los intereses de sus habitantes.

Finalmente este proyecto ejemplifica cómo la búsqueda de rentabilidad puede ser orientada hacia la mejora de servicios de interés comunitario.

C) El caso Mar del Plata Juegos Deportivos Panamericanos '95 demostró que, una vez más se sostuvo la actitud de mirar hacia los paradigmas internacionales, sin embargo, estos no siempre resultan de fácil aplicación en una ciudad latinoamericana de porte medio, con problemas financieros y urbanos propios; y que la oportunidad presentada para encarar algunos problemas de este último tipo, fue desaprovechada.

Acordamos que: "...la ciudad es el ámbito donde se entrelazan relaciones sociales, económicas, poblacionales y recursos naturales, tareas políticas y el quehacer cultural $^{\prime 14}$. En ella se teje una nítida red de relaciones e interdependencias, se presentan los órdenes más complejos y es ahí donde principalmente se resuelven. Es la ciudad, el reflejo de como evolucionó y evoluciona una región, una sociedad o las fuerzas impulsoras de su transformación. Nos toca vivir la época de las urbanizaciones aceleradas y explosiones demográficas, de entrada y salida a una economía global, donde el verdadero capital es el conocimiento y la información, y aquel que los posea podrá ser quien tenga mayor capacidad de acción y de éxito.

En nuestro caso, según R. Fernández frente a la inviabilidad de llevar adelante una verdadera reforma urbana (necesaria para la ciudad), las instalaciones para los XII Juegos se concentran en un terreno irregular del borde de la ciudad... predio de excelente localización y accesibilidad ${ }^{15}$.

Hemos visto como el análisis realizado demuestra claramente que esta concepción disminuye la posibilidad de influencia de las actuaciones del Estado a nivel urbano, restringiendo los resultados a las intervenciones específicas.

14 SILBERFADEN, Daniel H., "La ciudad futura". Arquis Nro. 5. Universidad de Palermo. Buenos Aires. 1995.

15 FERNÁNDEZ, Roberto (Op. Cit).

Revista de Urbanismo N9 - Marzo de 2004 
http://revistaurbanismo.uchile.cl

En un contexto socio-político no tan promisorio para el desarrollo urbano, la arquitectura, reducida a un virtuoso aprovechamiento de condicionamientos en este caso, parece haber salido disciplinariamente airosa, ganando 'prestigio' cultural e incluso popular y reivindicando la necesidad de seguir generando equipamiento público. No parece tan poco rédito en este exasperante ultramercantil y privatista fin de siglo ${ }^{16 .}$

\section{Reflexiones de carácter general}

Los distintos caminos recorridos (en un trabajo de estas características), así como la variedad de teorías y ejemplos analizados (incluso en distintos momentos históricos) permiten, hoy, arrojar algunas reflexiones de carácter amplio, que trascienden el ámbito de los sectores estudiados, y comprenden a la ciudad en general.

Entendida la construcción de la ciudad como una compleja relación entre distintos actores, deviene necesaria la participación de un agente coordinador en este proceso (sea desde el Estado, o desde alguna organización intermedia), el cual con ciertos objetivos concretos, obtenga resultados que en definitiva redunden en beneficio de la ciudad. Más, si tomamos en cuenta por los casos analizados en nuestra ciudad, que las transformaciones más significativas son aquellas que surgen de la acción espontánea de actores privados, donde la intervención del Estado se visualiza como un mero acompañamiento jurídico (o legitimación) de los cambios producidos.

Ahora bien, según lo estudiado en el presente trabajo, para poder influir en dicho proceso de construcción el agente coordinador deberá poseer cierta aptitud que le permita conocer y dominar:

a).-las posibilidades, y los límites, que ofrece la normativa vigente, y los modos de actuar dentro de ella;

b).- las características del sector en cuestión, (su estado, aprovisionamiento de servicios, valores) y las repercusiones potenciales luego de intervenirlo;

c).- la caracterización de todos y cada uno de los actores involucrados; y

d).- la viabilidad política de llevar adelante el proyecto.

16 FERNANDEZ, Roberto, "Mar del Plata. Arquitectura si, urbanismo no". En Revista Arquis Nro. 6. Universidad de Palermo. Buenos Aires. 1995.

Revista de Urbanismo N9 - Marzo de 2004 
http://revistaurbanismo.uchile.cl

A su vez, no puede presentar una actitud neutral, sino, más bien, propositiva, cargada de ideas ${ }^{17}$, que permitan orientar los procesos espontáneos o generar otros nuevos, con la mirada puesta en el beneficio de toda la ciudad (sociedad).

De los aspectos citados, y sus variadas implicancias, surge la necesidad de entender al agente coordinador como a un grupo pluridisciplinar (con participación técnica diversa y representación de distintos sectores).

Atacar los problemas urbanos desde la perspectiva de una sola disciplina dejaría trunco el análisis y haría inconducente cualquier tipo de propuesta. Del mismo modo que atender solamente a algunos de los sectores involucrados generaría conflictos en su desarrollo y beneficiaría a estos en perjuicio de los demás.

Mar del Plata, verano de 2003.

\section{Bibliografía}

BOHIGAS, Oriol, "Una nueva Barcelona. Reflexiones sobre los últimos diez años". A\&V (Monografías de Arquitectura y Vivienda) Nro. 37. Madrid, 1992.

BOHIGAS/BUCHANAN/LAMPUGNANI Barcelona. Arquitectura y Ciudad (1980-1992). Barcelona. G. Gilli, 1991.

CASTELLS, Manuel, La ciudad informacional. Madrid, Alianza, 1995.

CASTELLS, Manuel, Crisis urbana y cambio social. México. Siglo XXI. 1987.

CIGNOLI, Alberto L., "Intervenciones en el Espacio Territorial". En Arquitectura No 8 año 5, ASAMP, Mar del Plata, 1987.

CLICHEVSKY, Nora y otros, Construcción y administración de la ciudad Latinoamericana. Buenos Aires. GEL, 1990.

ESQUIAGA, José María, "Entre el plan y el proyecto". En A\&V (Monografías de Arquitectura y Vivienda) Nro. 30. Madrid, 1991.

FERNÁNDEZ, Roberto, "Realismo fin de siglo". En Revista Summa + Nro. 13. Buenos Aires, 1995.

17 Un trabajo propio, que se encuentra en elaboración, aborda decididamente esta cuestión. 


\section{REVITA DE \\ URBANISMO \\ ISSN 0717-5051}

http://revistaurbanismo.uchile.cl

FERNÁNDEZ, Roberto, "Mar del Plata. Arquitectura si, urbanismo no". En Revista Arquis Nro. 6. Universidad de Palermo. Buenos Aires, 1995.

FOLIN, Marino, La ciudad del capital y otros escritos. México, G. Gilli, 1977.

GALEANO, Eduardo, Las venas abiertas de América Latina. Siglo XXI. Buenos Aires. 1986.

GARAY, Alfredo, "Repensando el Proyecto". En Revista Arquis Nro.1. Universidad de Palermo. Buenos Aires, 1994.

GARCIA-PELAYO, Manuel, Las transformaciones del Estado Contemporaneo. Madrid. Alianza Universidad, 1987.

GORELIK, Adrián Imaginarios urbanos e imaginación urbana. Ponencia. En BazarAmericano.com

GUIMARAES, Roberto, "El leviatán en extinción? Notas sobre reforma del Estado en América Latina". En Revista SITUACIÓN Nro. 1. junio 1996.

KOOLHAAS, Rem, "La ciudad genérica". En Dossier Ciudad. Beta_test/e.zine sobre arte, tecnología y medios, 2002.

KUHN, T.S.,La estructura de las revoluciones científicas. FCE. México. 1985.

LAMPUGNANI, Vittorio M., "La ciudad de la tolerancia. Sobre la construcción del presente". En A\&V (Monografías de Arquitectura y Vivienda) Nro. 22. 1990.

MARTORILL/BOHIGAS/MACKAY/PUIGDOMENECH, La villa Olímpica. Arquitectura. Parques. Puerto Deportivo. Barcelona. G.Gilli, 1991.

MARTORILL/BOHIGAS/MACKAY/PUIGDOMENECH, Transformación de un Frente Marítimo. Barcelona. La villa Olímpica. Barcelona. G.Gilli.,1988.

MONTANER, Josep M., "El ensanche litoral". A\&V (Monografías de Arquitectura y Vivienda) Nro.22. Madrid, 1990.

O’DONNELL, Guillermo, Apuntes para una Teoría del Estado. Doc. CEDES/ G.E.CLACSO Nro. 9, 1977.

O'DONNELL, Guillermo, Reflexiones sobre las tendencias generales de cambio en el estado burocrático-autoritario. Doc. CEDES/ G.E.CLACSO Nro. 1, 1975.

OSZLAK, Oscar, Estado y sociedad. Nuevas reglas de juego? En línea www.clad.org.ve OSZ PLA, Alberto, Estado y sociedad en el pensamiento Norte y Latinoamericano. 


\section{REVISTA DE

http://revistaurbanismo.vchile.cl

Cántaro. Bs.As., 1987.

RIVOIRA, Emilio, "Puerto Madero, antes que nada, gestión urbana". En Revista Arquis Nro. 1. Universidad de Palermo. Buenos Aires, 1994.

ROTOLO, Miguel, "Procesos de Transformación Urbana. Un aporte a las estrategias de regulación en las intervenciones urbanísticas". En I+A / Investigación + Acción. FAU UNMdP. Mar del Plata, 1994.

SANGUINETTI, Horacio, Dinámica Política. Curso de Derecho Político. Buenos Aires. Astrea, 1980.

SILBERFADEN, Daniel H., "La ciudad futura". Revista Arquis Nro. 5. Universidad de Palermo. Buenos Aires, 1995.

SOLÁ-MORALES, Ignasi, "De las plazas a los juegos". En A\&V (Monografías de Arquitectura y Vivienda) Nro. 22. Madrid, 1990 